\title{
Synthesis, Spectral Analysis and Biological Potency of Hydrazoneoxime Ligands Incorporating Pyrazolone Moiety and Their Metal Complexes
}

\author{
NURSABAH SARIKAVAKLI ${ }^{1 *}$, EMRAH KOÇ ${ }^{2}$, MESHARI M.H. ALJOHANI $^{3}$ \\ and SYED KHALID MUSTAFA** \\ 1,2Department of Chemistry, Faculty of Arts and Sciences, Aydın Adnan Menderes University, \\ Aydın-09010, Turkey. \\ ${ }^{3,4}$ Department of Chemistry, Faculty of Sciences, University of Tabuk, Kingdom of Saudi Arabia. \\ ${ }^{*}$ Corresponding author E-mail: nsarikavakli@adu.edu.tr, khalid.mustafa938@ gmail.com
}

http://dx.doi.org/10.13005/ojc/360626

(Received: October 09, 2020; Accepted: December 10, 2020)

\begin{abstract}
A modest attempt has been made for the synthesis of hydrazoneoxime ligands bearing pyrazolone group (1-4) and their successive metal complexes such as: 1(a-c), 2(a-c), 3(a-c) and 4(a-c). The precursor $(1 Z, 2 E)$-2-(hydroxyimino) ethanehydroximohydrazide $\left(\mathrm{GH}_{2}\right)$ was obtained through coupling of $(1 Z, 2 E)-\mathrm{N}$-hydroxy-2-(hydroxyimino) ethanimidoyl chloride and hydrazinium hydroxideto generate hydrazonoxime compounds bearing the pyrazolone group. The ligands (1-4) were reacted with $\mathrm{MX}_{2} \cdot \mathrm{nH}_{2} \mathrm{O}$, where $\mathrm{M}=\mathrm{Co}(\mathrm{II}), \mathrm{Ni}(\mathrm{II})$ and $\mathrm{Cu}(\mathrm{II})$ to obtain the successive metal coordinated compounds into good yields. The ligands and their metal complexes were investigated by using ${ }^{1} \mathrm{H}$ NMR, ${ }^{13} \mathrm{C}$ NMR, FT-IR, elemental analysis and magnetic susceptibility measurements. Tautomerism in the ligands is investigated spectroscopically and biological activities are evaluated as well. Finally, the findings of present study were found within good egreement with other workers.
\end{abstract}

Keywords: Pyrazolone, Hydrazoneoxime, Tautomerism, Metal complexes, Spectral studies, Biological activities.

\section{INTRODUCTION}

Pyrazolone is considered as an important component or structural unit which is present in various active compounds. Owing to its convenient synthesis as well asversatile biological applications particularly its comprehensive antiseptics, antitumor, antibacterial action ${ }^{1-4}$, pyrazolone and its complexes have acquired a significant consideration in coordination as well as in medicinal chemistry.
Pyrazolone derived product sare paramount kind of heterocyclic compounds that occurs in various drugs as well as in synthetic products ${ }^{5,6}$. These compounds show extraordinary analgesic ${ }^{7}$, antitubercular ${ }^{8}$, antifungal, antibacterial ${ }^{9}$, antiinflammatory ${ }^{10}$, antioxidant and antitumor activities ${ }^{11}$. Because of their facile synthesis and character is ticbiological activity, pyrazolone arrangement performs a significant function and reflects an effective

This is an Open Access article licensed under a Creative Commons license: Attribution 4.0 International (CC- BY). Published by Oriental Scientific Publishing Company @ 2018 
example for combinatorial and pharmaceutical chemistry. Furthermore, pyrazole derived products showed outstanding biological asset, for example, anti-microbial ${ }^{12}$, analgesic ${ }^{13}$, anti-inflammatory ${ }^{14}$ and anticancer activities ${ }^{15}$. This provided anenormous boost to explore for potentially active compounds having pyrazole substituents.

Here in, the derivatization of hydrazone oxime ligands bearing pyrazolone group (1-4) and their metal complexes1(a-c), 2(a-c), 3(a-c), 4(a-c) was reported. The ligands and its complexes were characterized by ${ }^{1} \mathrm{H}$ NMR, ${ }^{13} \mathrm{C}$ NMR, FT-IR spectroscopy, elemental analysis and magnetic susceptibility techniques. The proposed general structure of the ligand is given in scheme.1

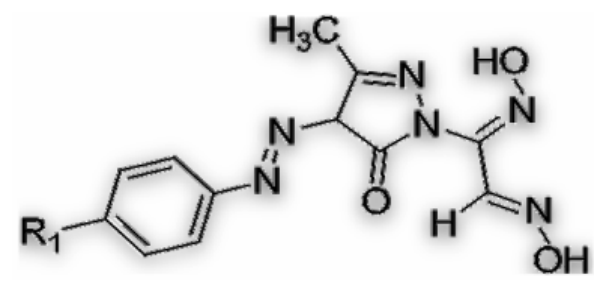

$\left[R_{1}: \mathrm{H}=1, \mathrm{p}-\mathrm{CH}_{3}=2, \mathrm{p}-\mathrm{NO}_{3}=3, \mathrm{p}-\mathrm{OCH}_{3}=4\right]$

Scheme 1. The moleculer formules of the ligands

EXPERIMENTAL

\section{Instruments and reagents}

Reagent sused in the experiment have been obtained from Sigma-Aldrich, Merck and Fluka and were handled as received. The melting point of the synthesised complexes and the ligands were varified and calculated by the Büchi SMP-20 apparatus using an open capillary method. For the calculation of FT-IR spectra, $\mathrm{KBr}$ discs on a Perkin Elmer Mattson 1000 spectrophotometer were used, ${ }^{1} \mathrm{H}$ and ${ }^{13} \mathrm{C}$ NMR spectra have been reported by Bruker-Spectrospin Avance DTX 400 Ultra-Shield in deuterated dimethyl sulphoxide (DMSO- $\left.d_{6}\right)$ and tetramethylsilane (TMS) used as an internal standard and chemical shifts considered in ppm. The Leco CHNS-932 analyzer used for the elemental analysis: and for the $\mathrm{pH}$ measurements, an Orion Expandable Ion Analyzer EA 940 was used. The melting point, colors, molecular weights, percentage of yield, molar conductance, magnetic susceptibilities (Sherwood Scientific) have been calculated and are given in the following sections.

Preparation of the ligands and their nomenclature The precursor $(1 Z, 2 E)$-2-(hydroxyimino) ethanehydroximohydrazide $\left(\mathrm{GH}_{2}\right)$ was prepared by the action of the $(1 Z, 2 E)$-N-hydroxy-2-(hydroxyimino) ethanimidoyl chloride ${ }^{16}$ and hydrazinium hydroxide. Since the parent compound, $\mathrm{GH}_{2}$ is unstable at normal temperature, so it was utilized without further purification or as it was received. Hydrazone oxime ligands bearing pyrazolone group (1-4) have beenprepared with the 5-pyrazolones as mentioned in the literature ${ }^{17}$ and characterized their structuresby using spectral techniques.

The IUPAC name of the ligands may be given as 2-[(1Z,2E)-N-hydrokis-2-(hydrokisimino) etanimidoil]-5-metil-4-[(Z)-fenildia zenil]-2,4dihidro-3H-pirazol-3-on(1),2-[(1Z,2E)-N-hydrokis-2(hydrokisimino) etanimidoil]-5-metil-4-[(Z)-(4-nitrofenil) diazenil]-2,4-dihidro-3H-pirazol-3-on (2), 2-[(1Z,2E)$\mathrm{N}$-hydro kis-2-(hydrokisimino)etanimidoil]-4-[(Z)-(4metoksifenil)diazenil]-5-metil-2,4-dihidro-3H-pirazol3-on (3) and 2-[(1Z,2E)-N-hydrokis -2-(hydrokisimino) etanimidoil]-5-metil-4-[(Z)-(4-metilfenil)diazenil]2,4-dihidro-3H-pirazol-3-on (4). The preparation procedures were followed as given in theliterature ${ }^{18-20}$. For the synthesis of (1-4) the common route follows as given in the Scheme 2 .

\section{General procedure of synthesis Synthesis of hydrazoneoxime ligands bearing pyrazolone group(1-4)}

Aniline derivatives $10 \mathrm{mmol}(0.69 \mathrm{~g}$, aniline; $1.38 \mathrm{~g}$, 4-nitroaniline; $1.23 \mathrm{~g}$, p-anisidine; or $1.04 \mathrm{~g}, \mathrm{p}$-toluidine respectively) were taken and dissolved in a mixture of 1:1 ratio of glacial acetic acid and concentrated hydrochloric acid $(20 \mathrm{~mL})$ and cooled the solution upto $0-5^{\circ} \mathrm{C}$. Sodium nitrite $(0.69 \mathrm{~g}$ or $0.01 \mathrm{~mol})$ was taken and dissolved in $10 \mathrm{~mL}$ water and then added dropwise to the above-prepared solution mixture with vigorous stirring for nearly one hour to maintain the solution temperature between $0-5^{\circ} \mathrm{C}$. The obtained diazonium solution has been mixed in aliquots for $30 \mathrm{~min}$ to thesolution of 2-[(1Z,2Z)-N-hydrokis-2(hydrokisimino)etanimidoil]-5-metil-2,4-dihidro-3Hpirazol-3-on $(0.76 \mathrm{~g}$ or $0.01 \mathrm{~mol})$ in $10 \mathrm{~mL}$ of ethyl alcohol and stirred vigorously, added the $\mathrm{NaOH}$ solution for maintaining the $\mathrm{pH}$ level between ${ }^{7-8}$. The mixture has bee stirred for $2 \mathrm{~h}$ and maintain the solution temperature between $0-5^{\circ} \mathrm{C}$. The obtained product was separated by means of diluting with water $(50 \mathrm{~mL})$, filtered, washed several times with distilled water and finally dried. 


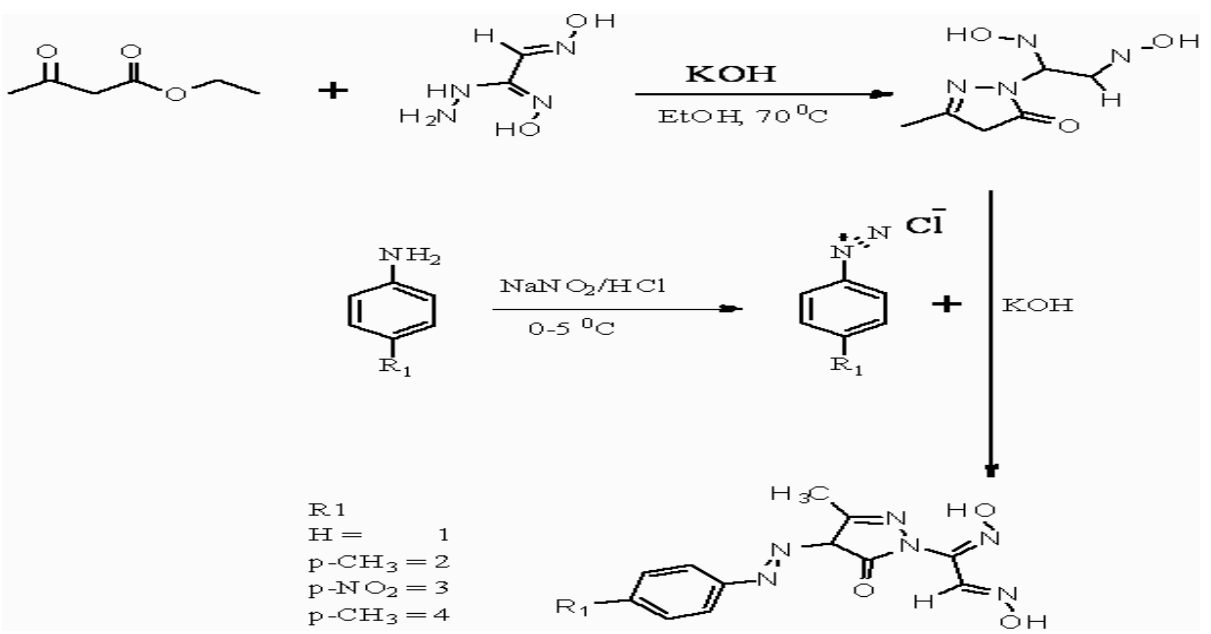

Scheme 2. General route for the synthesis of ligands (1-4)

2-[(1Z,2E)-N-hydrokis-2-(hydrokisimino) etanimidoil]-5-metil-4-[(Z)-fenildiazenil]-2,4dihidro-3H-pirazol-3-on(1)

The analytical and physical properties of the ligand (1) and its metal complexes (1a-1c) are provided in Table 1. Appearance: yellow powder; Yield=87\%, m.p. (decomp.) $165^{\circ} \mathrm{C}$; The reaction scheme is given in Scheme 2. The compound is soluble in commonly used solvents like $\mathrm{CH}_{2} \mathrm{Cl}_{2}, \mathrm{CHCl}_{2}, \mathrm{DMF}, \mathrm{EtOH}$ and DMSO. Anal. Calcd. for $\mathrm{C}_{12} \mathrm{H}_{12} \mathrm{~N}_{6} \mathrm{O}_{3}\left(288.27 \mathrm{~g} \mathrm{~mol}^{-1}\right): 50.00 \%$ C; $4.20 \% \mathrm{H} ; 29.15 \%$ N, Found: $50.60 \%$ C; $4.40 \% \mathrm{H}$; $29.21 \% \mathrm{~N}$. The infrared spectral of the ligand (1) is given in Table 2. FT-IR (KBr, $\left.v_{\max } / \mathrm{cm}^{-1}\right): 3271(\mathrm{~N}-\mathrm{H})$,
3681(O-H), $2970\left(\mathrm{C}-\mathrm{H}_{\text {Arom. }}\right), 2844\left(\mathrm{C}-\mathrm{H}_{\text {Aliph. }}\right), 1598$ $1664\left(\mathrm{C}=\mathrm{N}_{\text {Oxim }}\right), 1556\left(\mathrm{C}=\mathrm{N}_{\text {Hydr. }}\right), 1033(\mathrm{~N}-\mathrm{O}), 1484$ ve $1441\left(\mathrm{~N}-\mathrm{N}_{\text {Azo }}\right)$. The FT-IR spectrum of ligand (1) is given in Fig. 1. ${ }^{1} \mathrm{H}$ NMR peaks (DMSO-d ${ }_{6},(\delta)$ ppm): 2,18(s,3H, pyazolone-CH3); 7.17-7.57 (m,5H, Ar-H); $11.81 ; 12.20$ (d, 2H (OH); 7.84 (s, 2H, $\mathrm{CH}=\mathrm{NOH})$; 13.01 (s, $2 \mathrm{H}-\mathrm{CH}=\mathrm{N}-\mathrm{NH}) ; 11.81 ; 12.20$ (s, $4 \mathrm{H}(\mathrm{OH})$; $7.84 \mathrm{~s}, 2 \mathrm{H}(\mathrm{CH}=\mathrm{NOH}), 7.39 ; 7.40 ; 7.42 \mathrm{t}, 7.55 ; 7.57$ d, 7.17; 7.18; 7.20 t; 4H (Ar-H), 13.01 s, $2 \mathrm{H}(-\mathrm{CH}=\mathrm{N}-$ $\mathrm{NH}) \cdot{ }^{13} \mathrm{C}$ NMR peaks of the compound: $\left(\mathrm{CDCl}_{3}, \mathrm{TMS}, \delta\right.$ ppm):148.35(N-C=N-OH); 148.80(CH=N-OH); 156.99 $(\mathrm{C}=\mathrm{N}-\mathrm{N}) ; 12.03\left(\mathrm{CH}_{3}\right) ; 158.17(\mathrm{C}=\mathrm{N}-\mathrm{NH}) ; 159.76(\mathrm{C}=\mathrm{O})$; 129.96, 116.68, 126.05, 142.80 (Ar-C).
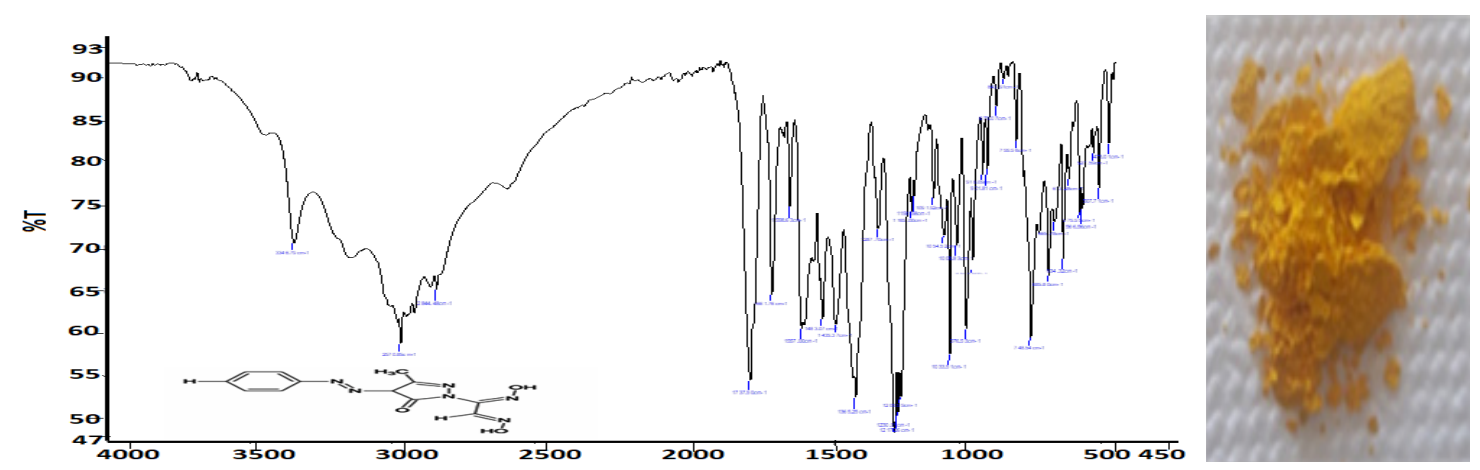

Fig. 1. FT-IR spectrum of ligand (1)

Table 1: Physical and analytical data of the hydrazoneoxime lıgand(1) bearıng pyrazolone group and its complexes

\begin{tabular}{|c|c|c|c|c|c|c|c|c|}
\hline \multirow[t]{2}{*}{ Compound } & \multirow{2}{*}{$\begin{array}{c}\text { Molecula } \\
\text { Composition }\end{array}$} & \multirow{2}{*}{$\begin{array}{l}\text { M.W. } \\
(\mathrm{g} / \mathrm{mol})\end{array}$} & \multirow[t]{2}{*}{ Apperance } & \multirow{2}{*}{$\begin{array}{l}\text { Melting Point } \\
(\mathrm{d})^{*}\left({ }^{\circ} \mathrm{C}\right)\end{array}$} & \multirow[t]{2}{*}{ Yield (\%) } & \multicolumn{3}{|c|}{ Calculated (Found)\% } \\
\hline & & & & & & C & $\mathrm{H}$ & $\mathrm{N}$ \\
\hline Ligand (1) & $\mathrm{C}_{12} \mathrm{H}_{12} \mathrm{~N}_{6} \mathrm{O}_{3}$ & 288,27 & Yellow & 165 & 87 & $50,00(50,60)$ & $4,20(4,40)$ & $29,15(29,21)$ \\
\hline Complex(1a) & $\mathrm{C}_{24} \mathrm{H}_{22} \mathrm{~N}_{12} \mathrm{O}_{6} \mathrm{Ni}$ & 633,21 & Red-brown & $>370^{*}$ & 52 & $45,52(45,60)$ & $3,50(3,43)$ & $26,54(26,47)$ \\
\hline Complex(1b) & $\mathrm{C}_{24} \mathrm{H}_{22} \mathrm{~N}_{12} \mathrm{O}_{6} \mathrm{Co}$ & 633,45 & Dark-brown & $230^{*}$ & 76 & $45,51(45,58)$ & $3,50(3,43)$ & $26,53(26,44)$ \\
\hline Complex(1c) & $\mathrm{C}_{24} \mathrm{H}_{22} \mathrm{~N}_{12} \mathrm{O}_{6} \mathrm{Cu}$ & 638,06 & Dark-brown & $193^{*}$ & 68 & $45,18(45,59)$ & $3,48(3,55)$ & $26,34(25,77)$ \\
\hline
\end{tabular}

$(d)^{\star}$ : decomposition 
Table 2: Infrared data for the ligand(1) and its complexes

\begin{tabular}{|c|c|c|c|c|c|c|c|c|c|c|}
\hline Compound & $v(\mathrm{O}-\mathrm{H})$ & $v(\mathrm{~N}-\mathrm{H})$ & $v(\mathrm{C}=\mathrm{O})$ & $v(\mathrm{C}=\mathrm{N})_{\text {oxim }}$ & $v(C=N)_{\text {hyd. }}$ & $v(\mathrm{CH})_{\text {Arom. }}$ & $v(\mathrm{CH})_{\text {Alip. }}$ & $v(\mathrm{~N}-\mathrm{N})$ & $v(\mathrm{~N}-\mathrm{O})$ & $v(\mathrm{OH}-\mathrm{O})$ \\
\hline Ligand (1) & $3681 w$ & $3271 w$ & $1667 s$ & $1598-1664 m$ & $1536-1556 \mathrm{~m}$ & $2970 m$ & $3088 m$ & $1484-1441 \mathrm{~m}$ & $1033 m$ & - \\
\hline Complex(1a) & $3271 \mathrm{~m}$ & $3271 \mathrm{~m}$ & $1667 w$ & $1594-1677 m$ & $1536-1556 \mathrm{~m}$ & $2970 m$ & $2844 m$ & $1449-1484 m$ & $1033 m$ & $1738 m$ \\
\hline Complex(1b) & $3271 \mathrm{~m}$ & $3271 \mathrm{~m}$ & $1667 w$ & $1594-1677 m$ & & $2970 m$ & $2844 m$ & $1449-1484 m$ & $1033 m$ & $1738 m$ \\
\hline Complex(1c) & $3168 \mathrm{~m}$ & $3168 \mathrm{~m}$ & $1655 \mathrm{~m}$ & $1594-1677 m$ & & $2970 m$ & $2864 m$ & $1449-1484 m$ & $1033 \mathrm{~m}$ & $1738 m$ \\
\hline
\end{tabular}

2-[(1Z,2E)-N-hydrokis-2-(hydrokisimino) etanimidoil]-5-metil-4-[(Z)-(4-nitrofenil) diazenil]2,4-dihidro-3H-pirazol-3-on (2)

Some important characteristics (analytical and physical) of the synthesized ligand (2) and its metal complexes (2a-2c) are givem in Table 3. Appearance: Dark brown powder; Yield $=75 \%$, m.p.(decomposition) $250^{\circ} \mathrm{C}$; For the synthesis of the compound (2), reaction arrangements are illustrated in Scheme 2. This compound is soluble in commonly used solvents like $\mathrm{CH}_{2} \mathrm{Cl}_{2}, \mathrm{CHCl}_{2}$, DMF, EtOH and DMSO. Anal. Calcd. for $\mathrm{C}_{12} \mathrm{H}_{11} \mathrm{~N}_{7} \mathrm{O}_{5}$ (333.27 $\mathrm{g} \mathrm{mol}^{-1}$ ): 43.25\% C; 3.33\% H; $29.42 \%$ N, Found: $43.83 \%$ C; $3.40 \% \mathrm{H} ; 29.35 \%$ N. The infrared spectral collected statistics of the ligand (2) are revealed in Table 4. FT-IR $\left(\mathrm{KBr}, v_{\text {max }} / \mathrm{cm}^{-1}\right)$ : $3271(\mathrm{~N}-\mathrm{H}), 3681(\mathrm{O}-\mathrm{H}), 2970\left(\mathrm{C}-\mathrm{H}_{\text {Arom }}\right), 2864$ $\left(\mathrm{C}-\mathrm{H}_{\text {Aliph. }}\right), 1609-1677\left(\mathrm{C}=\mathrm{N}_{\text {Oxim }}\right), 1543\left(\mathrm{C}=\mathrm{N}_{\mathrm{Hydr}}\right)$, 1033(N-O), 1506 ve $1483\left(\mathrm{~N}-\mathrm{N}_{\mathrm{Azo}}\right), 1417$ ve 1376 $\left(-\mathrm{NO}_{2}\right)$. The FT-IR spectrum of ligand (2) is given in Fig. 2. ${ }^{1} \mathrm{H}$ NMR peaks (DMSO- $d_{6}$, $(\delta)$ ppm): 2,20 (s, 3H, pyazolone-CH3); 7.68-7.78 (m,4H, Ar-H); 11.84; 12.22 (d, 2H (OH); 7,85 (s, 2H, CH=NOH); 13.03 (s, $2 \mathrm{H}-\mathrm{CH}=\mathrm{N}-\mathrm{NH}) \cdot{ }^{13} \mathrm{C}$ NMR peaks of the compound: $\left(\mathrm{CDCl}_{3}, \mathrm{TMS},(\delta) \mathrm{ppm}\right): 149.18(\mathrm{~N}-\mathrm{C}=\mathrm{N}-$ $\mathrm{OH}) ; 147.47(\mathrm{CH}=\mathrm{N}-\mathrm{OH}) ; 157.40(\mathrm{C}=\mathrm{N}-\mathrm{N}) ; 12.07$ $\left(\mathrm{CH}_{3}\right) ; 156.19(\mathrm{C}=\mathrm{N}-\mathrm{NH}) ; 158.05(\mathrm{C}=\mathrm{O}) ; 116.89$, 125.86, 129.41, 136.99 (Ar-C).

Table 3: Physical and analytical data of the hydrazoneoxime lıgand (2) bearıng pyrazolone group and its complexes

\begin{tabular}{|c|c|c|c|c|c|c|c|c|}
\hline \multirow[t]{2}{*}{ Compound } & \multirow{2}{*}{$\begin{array}{c}\text { Molecula } \\
\text { Composition }\end{array}$} & \multirow{2}{*}{$\begin{array}{l}\text { M.W. } \\
(\mathrm{g} / \mathrm{mol})\end{array}$} & \multirow[t]{2}{*}{ Apperance } & \multirow{2}{*}{$\begin{array}{l}\text { Melting Point } \\
\qquad(\mathrm{d})^{*}\left({ }^{\circ} \mathrm{C}\right)\end{array}$} & \multirow[t]{2}{*}{ Yield(\%) } & \multicolumn{3}{|c|}{ Calculated (Found)\% } \\
\hline & & & & & & $\mathrm{C}$ & $\mathrm{H}$ & $\mathrm{N}$ \\
\hline Ligand (2) & $\mathrm{C}_{12} \mathrm{H}_{11} \mathrm{~N}_{7} \mathrm{O}_{5}$ & 333,26 & Light-brown & 250 & 75 & $43,25(43,83)$ & $3,33(3,40)$ & $29,42(29,35)$ \\
\hline Complex(2a) & $\mathrm{C}_{24} \mathrm{H}_{20} \mathrm{~N}_{14} \mathrm{O}_{10} \mathrm{Ni}$ & 723,21 & Red-brown & $240 *$ & 80 & $39,86(39,93)$ & $2,79(2,85)$ & $27,12(27,69)$ \\
\hline Complex(2b) & $\mathrm{C}_{24} \mathrm{H}_{20} \mathrm{~N}_{14} \mathrm{O}_{10} \mathrm{C}_{0}$ & 723,45 & Dark-brown & $200^{*}$ & 76 & $39,85(39,27)$ & $2,79(3,34)$ & $27,11(27,67)$ \\
\hline Complex(2c) & $\mathrm{C}_{24} \mathrm{H}_{20} \mathrm{~N}_{14} \mathrm{O}_{10} \mathrm{Cu}$ & 728,06 & Dark-brown & $195^{*}$ & 65 & $39,59(39,99)$ & $2,77(2,83)$ & $26,93(26,99)$ \\
\hline
\end{tabular}

$(d)^{\star}$ : decomposition
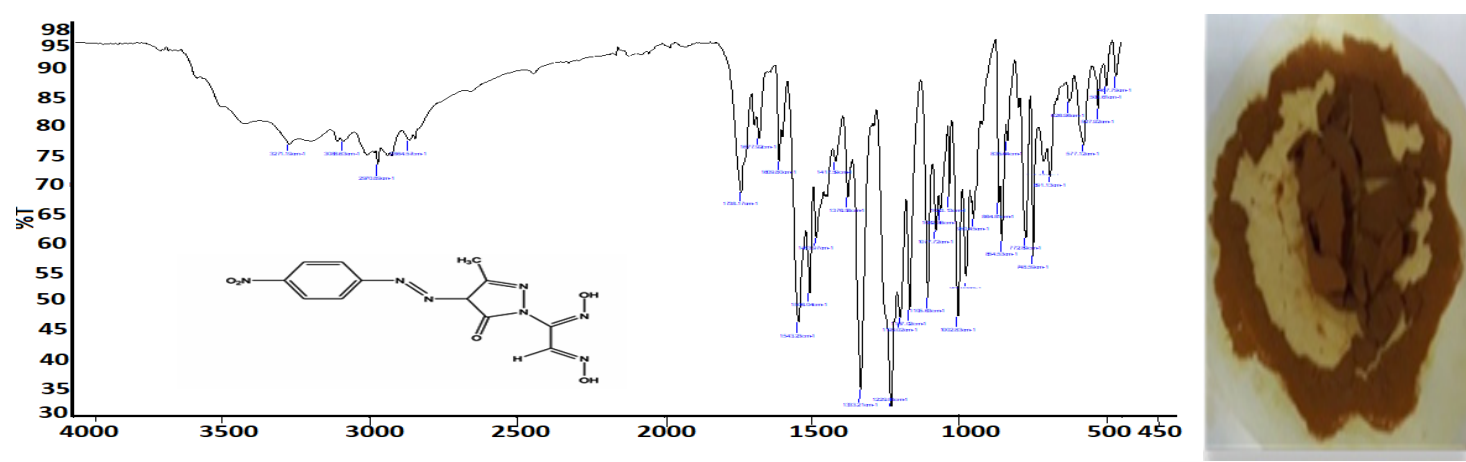

Fig. 2. FT-IR spectrum of ligand (2)

Table 4: Infrared data for the ligand (2) and its complexes

\begin{tabular}{ccccccccccccccc}
\hline Compound & $v(\mathrm{O}-\mathrm{H})$ & $v(\mathrm{~N}-\mathrm{H})$ & $v(\mathrm{C}=\mathrm{O})$ & $v(\mathrm{C}=\mathrm{N})_{\text {oxim }}$ & $v(\mathrm{C}=\mathrm{N})_{\text {hyd. }}$ & $v(\mathrm{CH})_{\text {Arom. }}$ & $v(\mathrm{CH})_{\text {Alip. }}$ & $v(\mathrm{~N}-\mathrm{N})$ & $v(\mathrm{~N}-\mathrm{O})$ & $v(\mathrm{OH}-\mathrm{O})$ \\
\hline Ligand (2) & $3681 \mathrm{w}$ & $3271 \mathrm{w}$ & $1667 \mathrm{~s}$ & $1598-1664 \mathrm{~m}$ & $1536-1556 \mathrm{~m}$ & $2970 \mathrm{~m}$ & $3088 \mathrm{~m}$ & $1484-1441 \mathrm{~m}$ & $1033 \mathrm{~m}$ & - \\
Complex(2a) & $3271 \mathrm{~m}$ & $3271 \mathrm{~m}$ & $1667 \mathrm{w}$ & $1594-1677 \mathrm{~m}$ & $1536-1556 \mathrm{~m}$ & $2970 \mathrm{~m}$ & $2844 \mathrm{~m}$ & $1449-1484 \mathrm{~m}$ & $1033 \mathrm{~m}$ & $1738 \mathrm{~m}$ \\
Complex(2b) & $3271 \mathrm{~m}$ & $3271 \mathrm{~m}$ & $1667 \mathrm{w}$ & $1594-1677 \mathrm{~m}$ & $1536-1556 \mathrm{~m}$ & $2970 \mathrm{~m}$ & $2844 \mathrm{~m}$ & $1449-1484 \mathrm{~m}$ & $1033 \mathrm{~m}$ & $1738 \mathrm{~m}$ \\
Complex(2c) & $3168 \mathrm{~m}$ & $3168 \mathrm{~m}$ & $1655 \mathrm{~m}$ & $1594-1677 \mathrm{~m}$ & $1536-1556 \mathrm{~m}$ & $2970 \mathrm{~m}$ & $2864 \mathrm{~m}$ & $1449-1484 \mathrm{~m}$ & $1033 \mathrm{~m}$ & $1738 \mathrm{~m}$ \\
\hline
\end{tabular}


2-[(1Z,2E)-N-hydrokis-2-(hydrokisimino) etanimidoil]-4-[(Z)-(4-metoksifenil) diazenil]-5metil-2,4-dihidro-3H-pirazol-3-on (3)

Some important characteristics (analytical and physical) of the synthesized ligand (3) and its metal complexes (3a-3c) are given in Table 5. Appearance: Red powder; Yield $=83 \%$, m.p.(decomposition) $230^{\circ} \mathrm{C}$; For the synthesis of the compound (3), reaction arrangements are illustrated in Scheme 2. This compound is soluble in commonly used solvents like $\mathrm{CH}_{2} \mathrm{Cl}_{2}, \mathrm{CHCl}_{2}, \mathrm{DMF}, \mathrm{EtOH}$ and DMSO. Anal. Calcd. for $\mathrm{C}_{13} \mathrm{H}_{14} \mathrm{~N}_{6} \mathrm{O}_{4}\left(318.29 \mathrm{~g} \mathrm{~mol}^{-1}\right)$ : $49.06 \%$ C; $4.43 \% \mathrm{H} ; 26.40 \%$ N, Found: $49.13 \%$ C; $4.37 \% \mathrm{H} ; 26.48 \% \mathrm{~N}$. The infrared spectral collected statistics of the ligand (3) are revealed in Table 6 . FT-IR (KBr, $\left.v_{\text {max }} / \mathrm{cm}^{-1}\right)$ : $3235(\mathrm{~N}-\mathrm{H}), 3681(\mathrm{O}-\mathrm{H}), 2970$ $\left(\mathrm{C}-\mathrm{H}_{\text {Arom }}\right), 2844\left(\mathrm{C}-\mathrm{H}_{\text {Aliph. }}\right), 1595-1648\left(\mathrm{C}=\mathrm{N}_{\text {Oxim }}\right), 1553$ $\left(\mathrm{C}=\mathrm{N}_{\text {Hydr }}\right), 1033(\mathrm{~N}-\mathrm{O}), 1454$ ve $1474\left(\mathrm{~N}-\mathrm{N}_{\text {Azo }}\right), 1738$ $-\mathrm{C}=\mathrm{O}$. The FT-IR spectrum ofligand $(3)$ is can be seen in Fig. 3. ${ }^{1} \mathrm{H}$ NMR peaks (DMSO- $d_{6}$, $(\delta) \mathrm{ppm}$ ): $2.16(\mathrm{~s}, 3 \mathrm{H}$, pyazolone- $\mathrm{CH} 3) ; 6.98-7.52(\mathrm{~m}, 4 \mathrm{H}, \mathrm{Ar}-\mathrm{H})$; 12.17-12.21 (d, 2H (OH); 7.83 (s, 2H, $\mathrm{CH}=\mathrm{NOH})$; 13.07 (s, $2 \mathrm{H}-\mathrm{CH}=\mathrm{N}-\mathrm{NH}) ; 3,74\left(\mathrm{~s}, 3 \mathrm{H}-\mathrm{OCH}_{3}\right.$. ${ }^{13} \mathrm{C}$ NMR peaks of the compound: $\left(\mathrm{CDCl}_{3}\right.$, TMS, $(\delta)$ ppm): $148.62(\mathrm{~N}-\mathrm{C}=\mathrm{N}-\mathrm{OH}) ; 144.97(\mathrm{CH}=\mathrm{N}-\mathrm{OH})$; $157.97(\mathrm{C}=\mathrm{N}-\mathrm{N}) ; 12.04\left(\mathrm{CH}_{3}\right) ; 157.26(\mathrm{C}=\mathrm{N}-\mathrm{NH})$; $158.35(\mathrm{C}=\mathrm{O}) ; 115.28,118.28,148.62,135.27$ (Ar-C); $55.89\left(\mathrm{OCH}_{3}\right)$.

Table 5: Physical and analytical data of the hydrazoneoxime lıgand(3) bearing pyrazolone group and its complexes

\begin{tabular}{|c|c|c|c|c|c|c|c|c|c|c|c|}
\hline \multirow[t]{2}{*}{ Compound } & \multirow{2}{*}{$\begin{array}{c}\text { Molecula } \\
\text { Composition }\end{array}$} & \multirow{2}{*}{$\begin{array}{l}\text { M.W. } \\
\text { (g/mol) }\end{array}$} & \multirow[t]{2}{*}{ Apperance } & \multirow{2}{*}{$\begin{array}{l}\text { Melting Point } \\
\qquad(\mathrm{d})^{*}\left({ }^{\circ} \mathrm{C}\right)\end{array}$} & \multirow[t]{2}{*}{ Yield(\%) } & \multicolumn{6}{|c|}{ Calculated (Found)\% } \\
\hline & & & & & & & $\mathrm{C}$ & & $\mathrm{H}$ & & $\mathrm{N}$ \\
\hline Ligand (3) & $\mathrm{C}_{13} \mathrm{H}_{14} \mathrm{~N}_{6} \mathrm{O}_{4}$ & 318,29 & Red & 230 & 83 & 49,06 & $(49,13)$ & 4,43 & $(4,37)$ & 26,40 & $(26,48)$ \\
\hline Complex(3a) & $\mathrm{C}_{26} \mathrm{H}_{26} \mathrm{~N}_{12} \mathrm{O}_{8} \mathrm{Ni}$ & 693,6 & Red-brown & $>370^{*}$ & 78 & 45,05 & $(45,13)$ & 3,78 & $(3,72)$ & 24,25 & $(24,32)$ \\
\hline Complex(3b) & $\mathrm{C}_{26} \mathrm{H}_{26} \mathrm{~N}_{12} \mathrm{O}_{8} \mathrm{Co}$ & 693,50 & Dark-brown & $>350^{*}$ & 65 & 45,03 & $(45,09)$ & 3,78 & $(3,72)$ & 24,24 & $(24,17)$ \\
\hline Complex(3c) & $\mathrm{C}_{26} \mathrm{H}_{26} \mathrm{~N}_{12} \mathrm{O}_{8} \mathrm{Cu}$ & 698,12 & Dark-brown & $196^{*}$ & 68 & 44,73 & $(44,79)$ & 3,75 & $(3,68)$ & 24,08 & $(24,66)$ \\
\hline
\end{tabular}

$(d)^{\star}$ : decomposition
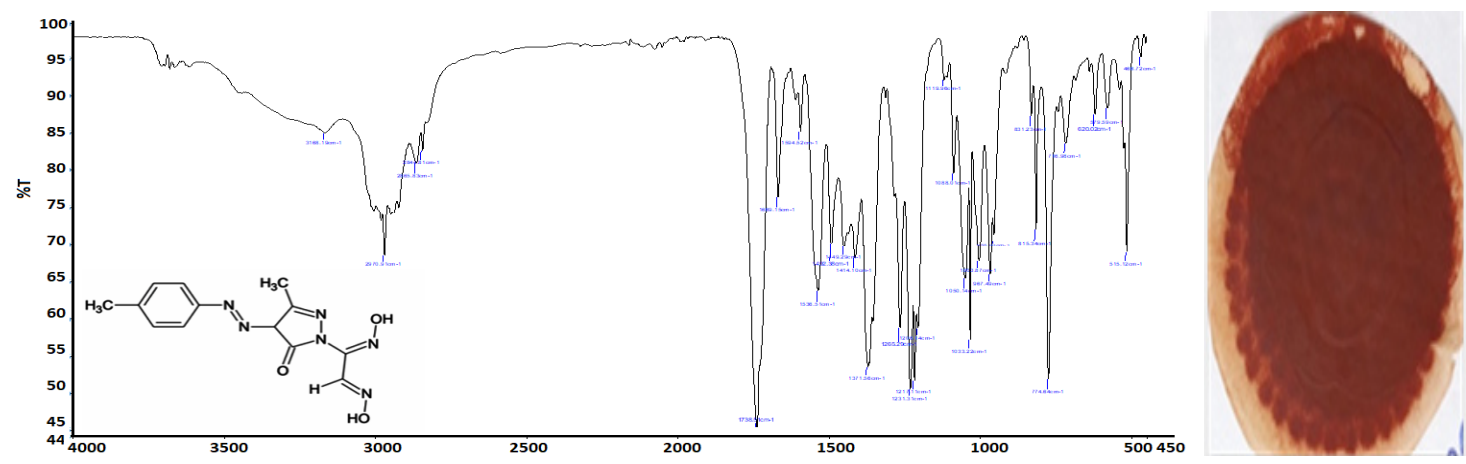

Fig. 3. FT-IR spectrum of ligand (3)

$2-[(1 Z, 2 E)-\mathrm{N}-$ hydrokis-2-(hydrokisimino) etanimidoil]-5-metil-4-[(Z)-(4-metilfenil) diazenil]2,4-dihidro-3H-pirazol-3-on (4)

Some important characteristics (analytical and physical) of thesynthesized ligand (4) and more (4a-4c) it's metal complexes are collected in Table 7. Appearance: Light brown powder: Yield $=76 \%$, m.p. (decomposition) $243^{\circ} \mathrm{C}$; For the synthesis of the compound (4), reaction arrangements are illustrated in Scheme 2. This compoundis soluble in commonly used solvents like $\mathrm{CH}_{2} \mathrm{Cl}_{2}, \mathrm{CHCl}_{2}$, DMF, EtOH and DMSO. Anal. Calcd. for $\mathrm{C}_{13} \mathrm{H}_{14} \mathrm{~N}_{6} \mathrm{O}_{3}$ (302.29 $\mathrm{g} \mathrm{mol}^{-1}$ ): $51.65 \% \mathrm{C} ; 4.67 \% \mathrm{H} ; 27.80 \% \mathrm{~N}$, Found: $51.70 \% \mathrm{C} ; 4.67 \% \mathrm{H} ; 27.80 \% \mathrm{~N}$. The infrared spectral collected statisticsof the ligand (4) are revealed in Table 8. FT-IR $\left(\mathrm{KBr}, v_{\max } / \mathrm{cm}^{-1}\right): 3168$ $(\mathrm{N}-\mathrm{H}), 3681(\mathrm{O}-\mathrm{H}), 2970\left(\mathrm{C}-\mathrm{H}_{\text {Arom. }}\right), 2844\left(\mathrm{C}-\mathrm{H}_{\text {Aliph. }}\right)$, 1594-1669 ( $\left.\mathrm{C}=\mathrm{N}_{\text {Oxim. }}\right), 1536\left(\mathrm{C}=\mathrm{N}_{\text {Hydr. }}\right), 1033(\mathrm{~N}-\mathrm{O})$, 1492 ve $1449\left(\mathrm{~N}-\mathrm{N}_{\text {Azo. }}\right), 1738-\mathrm{C}=\mathrm{O}, 2970-\mathrm{CH}_{3 \text { (phenil) }}$ ve $\mathrm{CH}_{3 \text { (methyl) }}$. The FT-IR spectrum of ligand (4) is given in Fig. 4. ${ }^{1} \mathrm{H}$ NMR peaks (DMSO- $d_{6},(\delta)$ ppm): 2.17 (s, 3H, pyazolone-CH3); 7.18-7.46 (m, $4 \mathrm{H}, \mathrm{Ar}-\mathrm{H}) ; 11.76 ; 12.18$ (d, 2H (OH); 7,83 (s, 2H, $\mathrm{CH}=\mathrm{NOH}$ ); 13.05 (s, 2H -CH=N-NH); 2.27 (s, 3H $-\mathrm{CH}_{3 \text { (fenil) }}{ }^{13} \mathrm{C}$ NMR peaks of the compound: $\left(\mathrm{CDCl}_{3}\right.$,

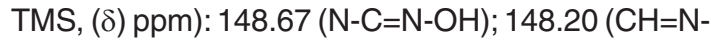
$\mathrm{OH}) ; 158.30(\mathrm{C}=\mathrm{N}-\mathrm{N}) ; 12.02\left(\mathrm{CH}_{3}\right) ; 157.12(\mathrm{C}=\mathrm{N}-$ $\mathrm{NH}) ; 160.64(\mathrm{C}=\mathrm{O}) ; 116.69,126.24,130.42,135.68$ (Ar-C); $20.97\left(\mathrm{CH}_{3}\right)$. 
Table 6: Infrared data for the ligand(3) and its complexes

\begin{tabular}{ccccccccccc}
\hline Compound & $v(\mathrm{O}-\mathrm{H})$ & $v(\mathrm{~N}-\mathrm{H})$ & $v(\mathrm{C}=\mathrm{O})$ & $v(\mathrm{C}=\mathrm{N})_{\text {oxim }}$ & $v(\mathrm{C}=\mathrm{N})_{\text {hyd. }}$ & $v(\mathrm{CH})_{\text {Arom. }}$ & $v(\mathrm{CH})_{\text {Alip. }}$ & $v(\mathrm{~N}-\mathrm{N})$ & $v(\mathrm{~N}-\mathrm{O})$ & $v(\mathrm{OH}-\mathrm{O})$ \\
\hline Ligand (3) & $3681 \mathrm{w}$ & $3271 \mathrm{w}$ & $1667 \mathrm{~s}$ & $1598-1664 \mathrm{~m}$ & $1536-1556 \mathrm{~m}$ & $2970 \mathrm{~m}$ & $3088 \mathrm{~m}$ & $1484-1441 \mathrm{~m}$ & $1033 \mathrm{~m}$ & - \\
Complex(3a) & $3271 \mathrm{~m}$ & $3271 \mathrm{~m}$ & $1667 \mathrm{w}$ & $1594-1677 \mathrm{~m}$ & $1536-1556 \mathrm{~m}$ & $2970 \mathrm{~m}$ & $2844 \mathrm{~m}$ & $1449-1484 \mathrm{~m}$ & $1033 \mathrm{~m}$ & $1738 \mathrm{~m}$ \\
Complex(3b) & $3271 \mathrm{~m}$ & $3271 \mathrm{~m}$ & $1667 \mathrm{w}$ & $1594-1677 \mathrm{~m}$ & & $2970 \mathrm{~m}$ & $2844 \mathrm{~m}$ & $1449-1484 \mathrm{~m}$ & $1033 \mathrm{~m}$ & $1738 \mathrm{~m}$ \\
Complex(3c) & $3168 \mathrm{~m}$ & $3168 \mathrm{~m}$ & $1655 \mathrm{~m}$ & $1594-1677 \mathrm{~m}$ & & $2970 \mathrm{~m}$ & $2864 \mathrm{~m}$ & $1449-1484 \mathrm{~m}$ & $1033 \mathrm{~m}$ & $1738 \mathrm{~m}$ \\
\hline
\end{tabular}

Table 7: Physical and analytical data of the hydrazoneoxime lıgand(4) bearıng pyrazolone group and its complexes

\begin{tabular}{|c|c|c|c|c|c|c|c|c|}
\hline \multirow[t]{2}{*}{ Compound } & \multirow{2}{*}{$\begin{array}{c}\text { Molecula } \\
\text { Composition }\end{array}$} & \multirow{2}{*}{$\begin{array}{l}\text { M.W. } \\
(\mathrm{g} / \mathrm{mol})\end{array}$} & \multirow[t]{2}{*}{ Apperance } & \multirow{2}{*}{$\begin{array}{l}\text { Melting Point } \\
\qquad(\mathrm{d})^{\star}\left({ }^{\circ} \mathrm{C}\right)\end{array}$} & \multirow[t]{2}{*}{ Yield(\%) } & \multicolumn{3}{|c|}{ Calculated (Found) \% } \\
\hline & & & & & & C & $\mathrm{H}$ & $\mathrm{N}$ \\
\hline Ligand (4) & $\mathrm{C}_{13} \mathrm{H}_{14} \mathrm{~N}_{6} \mathrm{O}_{3}$ & 302,29 & Dark-yellow & 243 & 76 & $51,65(51,70)$ & $4,67(4,71)$ & $27,80(27,74)$ \\
\hline Complex(4a) & $\mathrm{C}_{26} \mathrm{H}_{26} \mathrm{~N}_{12} \mathrm{O}_{6} \mathrm{Ni}$ & 661,27 & Red-brown & $>370$ * & 72 & $47,23(47,16)$ & $3,96(3,89)$ & $25,42(25,36)$ \\
\hline Complex(4b) & $\mathrm{C}_{26} \mathrm{H}_{26} \mathrm{~N}_{12} \mathrm{O}_{6} \mathrm{Co}$ & 661,51 & Dark-brown & $230 *$ & 70 & $47,21(47,28)$ & $3,96(3,89)$ & $25,41(25,57)$ \\
\hline Complex(4c) & $\mathrm{C}_{26} \mathrm{H}_{26} \mathrm{~N}_{12} \mathrm{O}_{6} \mathrm{Cu}$ & 666,12 & Dark-brown & $195^{*}$ & 64 & $46,88(46,81)$ & $3,93(3,88)$ & $25,23(27,17)$ \\
\hline
\end{tabular}

$(d)^{\star}:$ decomposition

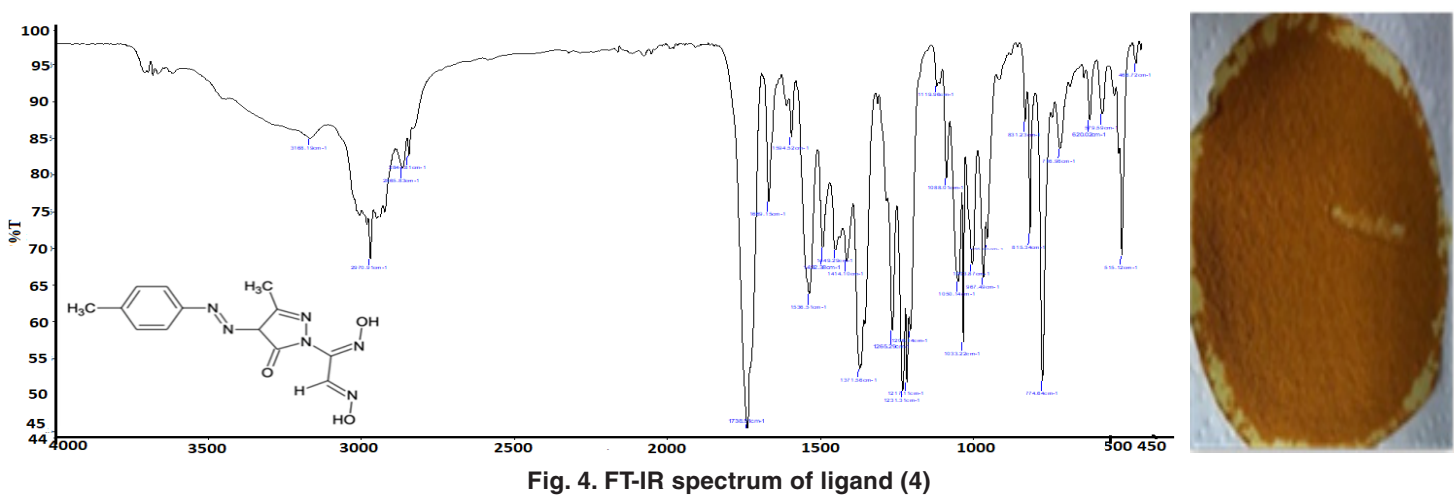

Table 8: Infrared data for the ligand(4) and its complexes

\begin{tabular}{lllllllllll}
\hline Compound & $v(\mathrm{O}-\mathrm{H})$ & $v(\mathrm{~N}-\mathrm{H})$ & $v(\mathrm{C}=\mathrm{O})$ & $v(\mathrm{C}=\mathrm{N})_{\text {oxim }}$ & $v(\mathrm{C}=\mathrm{N})_{\text {hyd. }}$ & $v(\mathrm{CH})_{\text {Arom. }}$ & $v(\mathrm{CH})_{\text {Alip. }}$ & $v(\mathrm{~N}-\mathrm{N})$ & $v(\mathrm{~N}-\mathrm{O})$ & $v(\mathrm{OH}-\mathrm{O})$ \\
\hline Ligand (4) & $3681 \mathrm{w}$ & $3271 \mathrm{w}$ & $1667 \mathrm{~s}$ & $1598-1664 \mathrm{~m}$ & $1536-1556 \mathrm{~m}$ & $2970 \mathrm{~m}$ & $3088 \mathrm{~m}$ & $1484-1441 \mathrm{~m}$ & $1033 \mathrm{~m}$ & - \\
Complex(4a) & $3271 \mathrm{~m}$ & $3271 \mathrm{~m}$ & $1667 \mathrm{w}$ & $1594-1677 \mathrm{~m}$ & $1536-1556 \mathrm{~m}$ & $2970 \mathrm{~m}$ & $2844 \mathrm{~m}$ & $1449-1484 \mathrm{~m}$ & $1033 \mathrm{~m}$ & $1738 \mathrm{~m}$ \\
Complex(4b) & $3271 \mathrm{~m}$ & $3271 \mathrm{~m}$ & $1667 \mathrm{w}$ & $1594-1677 \mathrm{~m}$ & $1536-1556 \mathrm{~m}$ & $2970 \mathrm{~m}$ & $2844 \mathrm{~m}$ & $1449-1484 \mathrm{~m}$ & $1033 \mathrm{~m}$ & $1738 \mathrm{~m}$ \\
Complex(4c) & $3168 \mathrm{~m}$ & $3168 \mathrm{~m}$ & $1655 \mathrm{~m}$ & $1594-1677 \mathrm{~m}$ & $1536-1556 \mathrm{~m}$ & $2970 \mathrm{~m}$ & $2864 \mathrm{~m}$ & $1449-1484 \mathrm{~m}$ & $1033 \mathrm{~m}$ & $1738 \mathrm{~m}$ \\
\hline
\end{tabular}

\section{Compound's Synthesis}

For the synthesis of all three complexes, a general method was used ${ }^{16}$. The $2 \mathrm{mmol}$ of the above ligands (1-4), were dissolved in $10 \mathrm{~mL}$ absolute ethyl alcohol at refluxing temperature. $\mathrm{A}$ solution of $\mathrm{NiCl}_{2} \cdot 6 \mathrm{H}_{2} \mathrm{O}$ or $\mathrm{CuCl}_{2} \cdot 2 \mathrm{H}_{2} \mathrm{O}$ or $\mathrm{CoCl}_{2} \cdot 6 \mathrm{H}_{2} \mathrm{O}$ $(1 \mathrm{mmol})$ with water $(15 \mathrm{~mL})$ is taken and then mixed dropwise over the ligand solution with vigorous stirring, a noticeable color change as well as a reduction in the $\mathrm{pH}$ value $(\sim 3.0-3.5)$ was observed. Sodium hydroxide (1\%) in water $(20 \mathrm{~mL})$ has been mixed to adjust the $\mathrm{pH} \sim 5-5.5$ and maintained the temperature of the reaction mixture nearly to room temperature. Mixtures have been stirred by the hour at $50^{\circ} \mathrm{C}$ on a water bath so as to ensure complete precipitation of complexes. After one hour the precipitated solid has been filtered, washed by means of hot ethanol $(3 \times 5 \mathrm{~mL})$ also dehydrated in vacuo, over anhydrous $\mathrm{CaCl}_{2}$. Proposed structures for the monomeric $\mathrm{Co}(\mathrm{II}), \mathrm{Ni}(\mathrm{II}) \mathrm{Cu}(\mathrm{II})$ as well as the ligands (1-4) are given in Scheme 3.

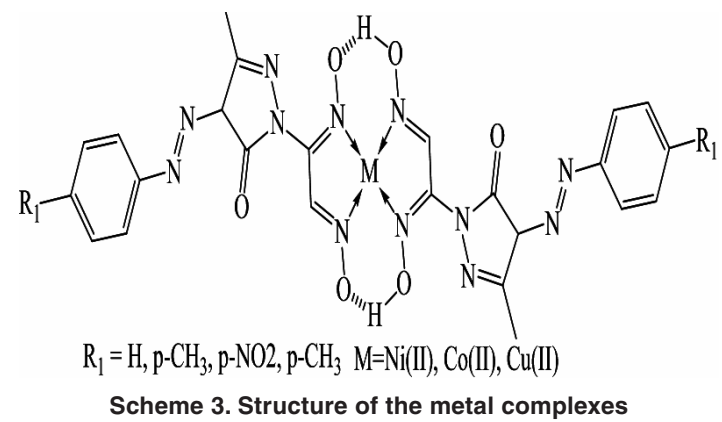


(1a) Appearance: Red-brown powder, Yield $=52 \%$, m.p. (decomp.) $>370^{\circ} \mathrm{C}$; This compoundis soluble in commonly used solvents like $\mathrm{CH}_{2} \mathrm{Cl}_{2}$, $\mathrm{CHCl}_{2}, \mathrm{DMF}, \mathrm{EtOH}$ and DMSO.Anal.Calcd. for $\mathrm{C}_{24} \mathrm{H}_{22} \mathrm{~N}_{12} \mathrm{O} \mathrm{O}_{6} \mathrm{Ni}\left(633.21 \mathrm{~g} \mathrm{~mol}^{-1}\right): 45.52 \% \mathrm{C} ; 3.50 \% \mathrm{H}$; $26.54 \%$ N, Found: $45.60 \%$ C; $3.43 \% \mathrm{H} ; 26.47 \% \mathrm{~N}$. FT-IR (KBr, $\left.v_{\text {max }} / \mathrm{cm}^{-1}\right): 3271(\mathrm{~N}-\mathrm{H}), 3681(\mathrm{O}-\mathrm{H}), 2970$ (C-HArom.), $2864\left(\mathrm{C}-\mathrm{H}_{\text {Aliph }}\right), 1609-1677\left(\mathrm{C}=\mathrm{N}_{\text {Oxim }}\right)$, $1543\left(\mathrm{C}=\mathrm{N}_{\text {Hydr. }}\right), 1033(\mathrm{~N}-\mathrm{O}), 1506$ ve $1483\left(\mathrm{~N}-\mathrm{N}_{\mathrm{AzO}}\right)$, 1417 ve $1376\left(-\mathrm{NO}_{2}\right)$.

(1b) Appearance: Dark brown powder, Yield $=76 \%$, m.p. (decomp.) $>230^{\circ} \mathrm{C}$; This compoundis soluble in commonly used solvents like $\mathrm{CH}_{2} \mathrm{Cl}_{2}, \mathrm{CHCl}_{2}$, DMF, EtOH and DMSO.Anal.Calcd. for $\mathrm{C}_{24} \mathrm{H}_{22} \mathrm{~N}_{12} \mathrm{O}_{6} \mathrm{Co}$ $\left(633,45 \mathrm{~g} \mathrm{~mol}^{-1}\right): 45.51 \% \mathrm{C} ; 3.50 \% \mathrm{H} ; 26.53 \% \mathrm{~N}$, Found: $45.58 \%$ C; $3.43 \% \mathrm{H} ; 26.44 \% \mathrm{~N}$.

(1c) Appearance: Dark brown powder, Yield $=68 \%$, m.p. (decomp.) $>193^{\circ} \mathrm{C}$; This compound is soluble in commonly used solvents like $\mathrm{CH}_{2} \mathrm{Cl}_{2}, \mathrm{CHCl}_{2}$, DMF, EtOH and DMSO.Anal.Calcd. for $\mathrm{C}_{24} \mathrm{H}_{22} \mathrm{~N}_{12} \mathrm{O}_{6} \mathrm{Cu}$ : $\left(638,06 \mathrm{~g} \mathrm{~mol}^{-1}\right): 45.18 \% \mathrm{C} ; 3.48 \% \mathrm{H} ; 26.34 \% \mathrm{~N}$, Found: $45.59 \% \mathrm{C} ; 3.55 \% \mathrm{H} ; 25.77 \% \mathrm{~N}$.

(2a) Appearance: Red-brown powder, Yield $=80 \%$, m.p.(decomp.) $>240^{\circ} \mathrm{C}$; This compound is soluble in commonly used solvents like $\mathrm{CH}_{2} \mathrm{Cl}_{2}, \mathrm{CHCl}_{2}$, DMF, EtOH and DMSO.Anal.Calcd. for $\mathrm{C}_{24} \mathrm{H}_{20} \mathrm{~N}_{14} \mathrm{O}_{10} \mathrm{Ni}$ $\left(723.21 \mathrm{~g} \mathrm{~mol}^{-1}\right): 39.86 \% \mathrm{C} ; 2.79 \% \mathrm{H} ; 27.11 \% \mathrm{~N}$, Found: $39.93 \%$ C; $2.85 \% \mathrm{H} ; 27.69 \% \mathrm{~N}$.

(2b) Appearance: Dark-brown powder, Yield $=76 \%$, m.p.(decomp.) $>200^{\circ} \mathrm{C}$; This compound is soluble in commonly used solvents like $\mathrm{CH}_{2} \mathrm{Cl}_{2}, \mathrm{CHCl}_{2}$, DMF, EtOH and DMSO.Anal.Calcd. for $\mathrm{C}_{24} \mathrm{H}_{20} \mathrm{~N}_{14} \mathrm{O}_{10} \mathrm{Co}$ (723.45 $\mathrm{g} \mathrm{mol}^{-1}$ ): $39.85 \% \mathrm{C} ; 2.79 \% \mathrm{H} ; 27.11 \% \mathrm{~N}$, Found: $39.27 \%$ C; $3.34 \% \mathrm{H} ; 27.67 \% \mathrm{~N}$.

(2c) Appearance: Dark-brown powder, Yield $=65 \%$, m.p.(decomp.) $>195^{\circ} \mathrm{C}$; This compound is soluble in commonly used solvents like $\mathrm{CH}_{2} \mathrm{Cl}_{2}, \mathrm{CHCl}_{2}$, DMF, EtOH and DMSO.Anal.Calcd. for $\mathrm{C}_{24} \mathrm{H}_{22} \mathrm{~N}_{12} \mathrm{O}_{6} \mathrm{Cu}$ $\left(728,06 \mathrm{~g} \mathrm{~mol}^{-1}\right): 39.59 \% \mathrm{C} ; 2.77 \% \mathrm{H} ; 26.93 \% \mathrm{~N}$, Found: $39.99 \%$ C; $2.83 \% \mathrm{H} ; 26.99 \% \mathrm{~N}$.

(3a) Appearance: Red-brown powder, Yield $=78 \%$, m.p.(decomp.) $>370^{\circ} \mathrm{C}$; This compound is soluble in commonly used solvents like $\mathrm{CH}_{2} \mathrm{Cl}_{2}, \mathrm{CHCl}_{2}$, DMF, EtOH and DMSO.Anal.Calcd. for $\mathrm{C}_{26} \mathrm{H}_{26} \mathrm{~N}_{12} \mathrm{O}_{8} \mathrm{Ni}$ (793,60 $\mathrm{g} \mathrm{mol}^{-1}$ ): $45.05 \% \mathrm{C} ; 3.78 \% \mathrm{H} ; 24.25 \% \mathrm{~N}$, Found: $45.13 \% \mathrm{C} ; 3.72 \% \mathrm{H} ; 24.32 \% \mathrm{~N}$. (3b) Appearance: Dark-brown powder, Yield $=65 \%$, m.p. (decomp.) $>350^{\circ} \mathrm{C}$; This compound is soluble in commonly used solvents like $\mathrm{CH}_{2} \mathrm{Cl}_{2}, \mathrm{CHCl}_{2}$, DMF, EtOH and DMSO.Anal.Calcd. for $\mathrm{C}_{26} \mathrm{H}_{26} \mathrm{~N}_{12} \mathrm{O}_{8} \mathrm{Co}$ (793,50 $\left.\mathrm{g} \mathrm{mol}^{-1}\right): 45.03 \% \mathrm{C} ; 3.78 \% \mathrm{H} ; 24.24 \% \mathrm{~N}$, Found: $45.09 \%$ C; $3.72 \% \mathrm{H} ; 24.17 \% \mathrm{~N}$.

(3c) Appearance: Dark-brown powder, Yield $=68 \%$, m.p.(decomp.) $>196^{\circ} \mathrm{C}$; This compound is soluble in commonly used solvents like $\mathrm{CH}_{2} \mathrm{Cl}_{2}, \mathrm{CHCl}_{2}$, DMF, EtOH and DMSO.Anal.Calcd. for $\mathrm{C}_{26} \mathrm{H}_{26} \mathrm{~N}_{12} \mathrm{O}_{8} \mathrm{Cu}$ $\left(798,12 \mathrm{~g} \mathrm{~mol}^{-1}\right): 44,73 \% \mathrm{C} ; 3,68 \% \mathrm{H} ; 24,08 \% \mathrm{~N}$, Found: $44,79 \%$ C; $3,68 \% \mathrm{H} ; 24,66 \% \mathrm{~N}$.

(4a) Appearance: Red-brown powder, Yield $=72 \%$, m.p. (decomp.) $>370^{\circ} \mathrm{C}$; This compound is soluble in commonly used solvents like $\mathrm{CH}_{2} \mathrm{Cl}_{2}, \mathrm{CHCl}_{2}$, DMF, EtOH and DMSO.Anal.Calcd. for $\mathrm{C}_{24} \mathrm{H}_{20} \mathrm{~N}_{14} \mathrm{O}_{10} \mathrm{Ni}$ (661, $\left.27 \mathrm{~g} \mathrm{~mol}^{-1}\right): 47.23 \% \mathrm{C} ; 3.96 \% \mathrm{H} ; 25.42 \% \mathrm{~N}$, Found: $45.16 \% \mathrm{C} ; 3.89 \% \mathrm{H} ; 25.36 \% \mathrm{~N}$.

(4b) Appearance: Dark-brown powder, yield $(70 \%)$, m.p.(decomp.) $>230^{\circ} \mathrm{C}$; This compound is soluble in commonly used solvents like $\mathrm{CH}_{2} \mathrm{Cl}_{2}, \mathrm{CHCl}_{2}$, DMF, EtOH and DMSO.Anal.Calcd. for $\mathrm{C}_{26} \mathrm{H}_{26} \mathrm{~N}_{12} \mathrm{O}_{8} \mathrm{Co}$ $\left(661,51 \mathrm{~g} \mathrm{~mol}^{-1}\right): 47.21 \% \mathrm{C} ; 3.96 \% \mathrm{H} ; 25.42 \% \mathrm{~N}$, Found: $47.28 \% \mathrm{C} ; 3.89 \% \mathrm{H} ; 25.57 \% \mathrm{~N}$.

(4c) Appearance: Dark-brown powder, Yield $=64 \%$, m.p. (decomp.) $>195^{\circ} \mathrm{C}$; This compound is soluble in commonly used solvents like $\mathrm{CH}_{2} \mathrm{Cl}_{2}, \mathrm{CHCl}_{2}$, DMF, EtOH and DMSO.Anal.Calcd. for $\mathrm{C}_{26} \mathrm{H}_{26} \mathrm{~N}_{12} \mathrm{O}_{8} \mathrm{Cu}$ (666, $12 \mathrm{~g} \mathrm{~mol}^{-1}$ ): $46.88 \% \mathrm{C} ; 3.93 \% \mathrm{H} ; 25.23 \% \mathrm{~N}$, Found: $46.81 \% \mathrm{C} ; 3.88 \% \mathrm{H} ; 27.17 \% \mathrm{~N}$.

\section{In vitro antimicrobic activity}

The antimicrobic activity of the test compounds was evaluated by way of agar well diffusion method ${ }^{21-22} .0 .1 \mathrm{~mL}$ of the diluted inoculums (106 CFU/mL) of test organisms are taken and spread on NA/SDA (Nutrient agar/Sabouraud dextrose agar) plates. Wells of $6 \mathrm{~mm}$ diameter have been punctured into the agar medium and filled one byone with $150 \mathrm{~mL}$ of compound $(150 \mu \mathrm{g} / \mathrm{L})$ solvent blank and anantibiotic (chloramphenicol, $100 \mu \mathrm{g} / \mathrm{L}$ ) to which the test the sensitivity of bacteria. Fluconazole at the concentration of $100 \mu \mathrm{g} / \mathrm{L}$ has been applied for the monitor versus Candida albicans, Candida tropicalis and Candida glabrata. The plates were incubated for at $37^{\circ} \mathrm{C} 24$ hours. Antimicrobial activity was has been assessed in order to the zone of inhibition versus the test organism. 


\section{RESULTS AND DISCUSSION}

Spectral studies: structure of ligands and complexes Synthesis of new hydrazoneoxime ligands bearing pyrazolone group (1-4) that were prepared by reaction of $(1 Z, 2 E)-\mathrm{N}$-hydroxy-2-(hydroxyimino) ethanimidoyl chloride ${ }^{16-17}$ with hydrazinium hydroxide and their metal complexes1(a-c), 2(a-c), 3(a-c), 4(a-c)were reported (Scheme 2 and 3). The ligands used in this work were prepared using the literature mentioned elsewhere ${ }^{18-20}$. The atomic arrangement of the synthesized ligands(1-4) including their metal complexes were established on the basis of their elemental analysis, FI-TIR, ${ }^{1} \mathrm{H}$ NMR, ${ }^{13} \mathrm{C}$ NMR, along with the calculation of their magnetic susceptibility as well. The analytical and physical properties of the prepared ligands including their metal complexes be summarised in Tables 1,3,5, along with 7 .

The FT-IR spectrum related to the synthesized ligands(1-4) as well as metal complexes1(a-c), 2(a-c), 3(a-c) and 4(a-c). 1-4 and 1(a-c), 2(a-c), 3(a-c) and 4(a-c) exhibited a carbonyl (keto) band at $1655-1667 \mathrm{~cm}^{-1}$ and $\mathrm{NH}$ band (hydrazo) at 3168$3271 \mathrm{~cm}^{-1}$ as shown in the IR spectral data of the representative ligand (1) (Fig. 1). The IR frequencies of the representative ligand and its complexes are shown in Table 1. The IR spectral collected statistics of the synthesized metal complexes has to begiven into Tables 2,4,6 and 8. Such said values indicated that each complexes are exist in keto-hydrazo form $\left(T_{2}\right)$ as well as in solid-state. ${ }^{23-25}$

The ${ }^{1} \mathrm{H}$ NMR spectra of all ligands (1-4) in DMSO- $d_{6}$ showed a single peak lying between 2.16-2.20 ppm corresponding to methyl protons (pyrazolone- $\mathrm{CH}_{3}$ ) at. The ${ }^{1} \mathrm{H}$ NMR spectral data of ligand (3) indicated a singlet for methoxy protons $\left(\mathrm{Ph}-\mathrm{OCH}_{3}\right)$ at $3.74 \mathrm{ppm}$ while another singlet for methyl protons $\left(\mathrm{Ph}^{\left.-\mathrm{CH}_{3}\right)}\right.$ at $2.27 \mathrm{ppm}$. The ${ }^{1} \mathrm{H}$ NMR spectra of all the ligands (1-4) showed multiple peak at between 6.98-7.78 ppm for aromatic protons (Ar-H). The ${ }^{1} \mathrm{H}$ NMR spectra of all ligands (1-4) also showed a single peaks for $(\mathrm{CH}=\mathrm{NOH})$ protons lying in the range of 7.83-7.85 ppm. They also exhibited a doublet for the characteristic oxime $\mathrm{OH}$ protons $(\mathrm{OH})$ in the range 11.76-12.22 ppm. Such chemical shifts have to be distinctive values, favour hydrazones and oximes, that help in to ascertain the proposed structure ${ }^{16,25}$.

The possible tautomeric forms of compounds (1-4) as indicated in Scheme 4. The compounds possibly be present in four likely tautomeric models i.e. keto-azo $\left(T_{1}\right.$ and $\left.T_{4}\right)$, keto-hydrazo $\left(T_{2}\right)$, as well asenol-azo $\left(T_{3}\right)$. In accordance with the literature, better stabilize automeric model exist in theketo-hydrazo form $\left(T_{2}\right)$. It may be due to the presence of the intramolecular $\mathrm{O}---\mathrm{H}$ bond. can show the stability of compounds in corresponding tautomeric form. The result is consistent with the literature ${ }^{23,26-29}$.

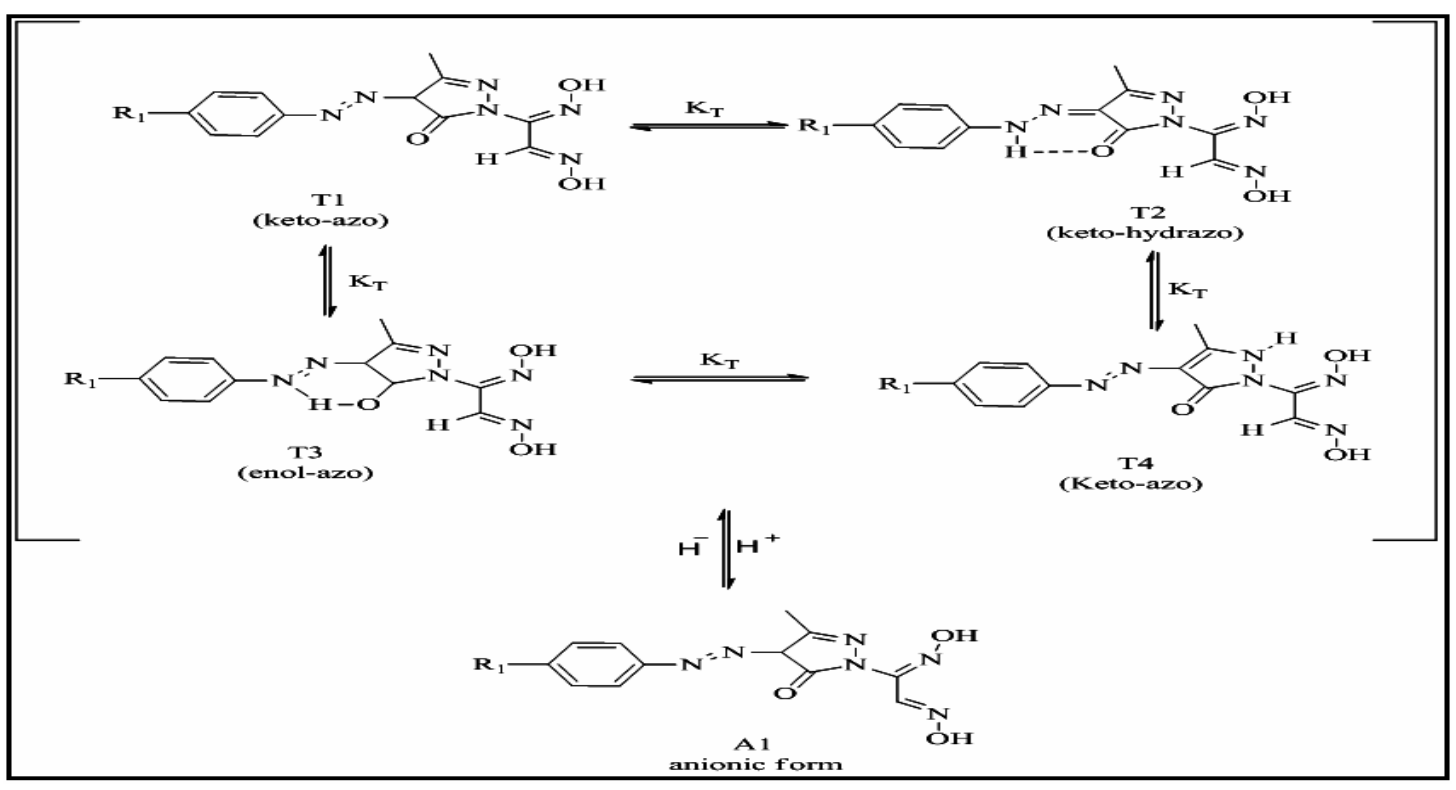

$\left[\mathrm{R}_{1}: \mathrm{H}, \mathrm{p}-\mathrm{CH}_{3}, \mathrm{p}-\mathrm{NO}_{2}, \mathrm{p}-\mathrm{OCH}_{3}\right]$

Scheme 4. Tautomeric structures and anionic forms of hydrazoneoxime ligands bearing pyrazolone group (1-4) 
The coordination compounds of nickel(II), copper(II) and cobalt(II) have been synthesized as per general methodology ${ }^{1,16}$ as discussed previously (Scheme 3). The metal ion and ligands react in 1:2 molar ratios, where the ligands are attached to metal by using its two $\mathrm{N}$ atoms, like almost all of vic-dioximes act. All of this coordinated compounds are colored, amorphous solids, stable at room temperature as well. The discussed complexes are insoluble in commonly used organic solvents, but are soluble in DMF and DMSO. The recommended structures of the synthesized coordination compounds has beendemonstrated in Scheme 3 that are supported by spectroscopic, FTIR spectral date and elemental analysis studies. As per the suggested structures, the complexes can have syn-or anti-conformation ${ }^{28}$. The magnetic moment ( $\mu$ eff) statistics further favoured the mononuclear structures of the coordinated complexes. The magnetic moment for the nickel(II) complexes are diamagnetic at room temperature, since it is expected due to the $d^{8}$ and $d^{10}$ electronic configurations $^{30}$. The magnetic moments ( $\mu$ eff) of the copper(II) complexes are 1.63, 1.76, 1.77 and $1.77 \mathrm{BM}$, respectively, so they are paramagnetic in nature. The cobalt(II) complexes of ligands are paramagnetic and their magnetic moments ( $\mu$ eff) are 2.05, 2.07, 2.08 and 2.09 BM, respectively. The lower figure possibly due to the anti ferromagnetic interaction between neighboring magnetic centers.

The another chemical environments will assign between two $(\mathrm{O} \cdot \bullet \cdot \mathrm{H}-\mathrm{O})$ bridge protons in the cis-form, at the same time one in the transform. Experience of the $\mathrm{H}$-bond $(\mathrm{O} \bullet \bullet \mathrm{H}-\mathrm{O})$ in the $\mathrm{IR}$ and ${ }^{1} \mathrm{H}$ NMR spectrum, appearing in single frequency in all cases, that indicates the nickel(II) complex is in the anti-form media. On the basis of the above experimental findings, the geometry of the nickel(II) and copper(II) and cobalt(II) complexes are recommended as square-planar ${ }^{1,16}$.

The possible tautomeric forms of new nickel(II) and copper(II) and cobalt(II) complexes 1(a-c), 2(a-c), 3(a-c) and 4(a-c) has to be explained in Scheme 5 The newly synthesized complexes possibly occurs into four probable tautomeric forms i.e. keto-azo $\left(T_{1}\right.$ and $\left.T_{4}\right)$, keto-hydrazo $\left(T_{2}\right)$ as well as in enol-azo $\left(T_{3}\right)$ form. As reported in the literature, utmost stabilized tautomeric form is a keto-hydrazo form $\left(\mathrm{T}_{2}\right)$ for azopyrazolone dyes. The complexes have an intramolecular hydrogen bonding $\mathrm{N}-\mathrm{H}$...O for keto-hydrazone $\left(\mathrm{T}_{2}\right)$ form and $\mathrm{O}-\mathrm{H}$...N for enol-azo $\left(\mathrm{T}_{3}\right)$ form. These results are consistent with the literature $\mathrm{e}^{23,26-29,31}$.

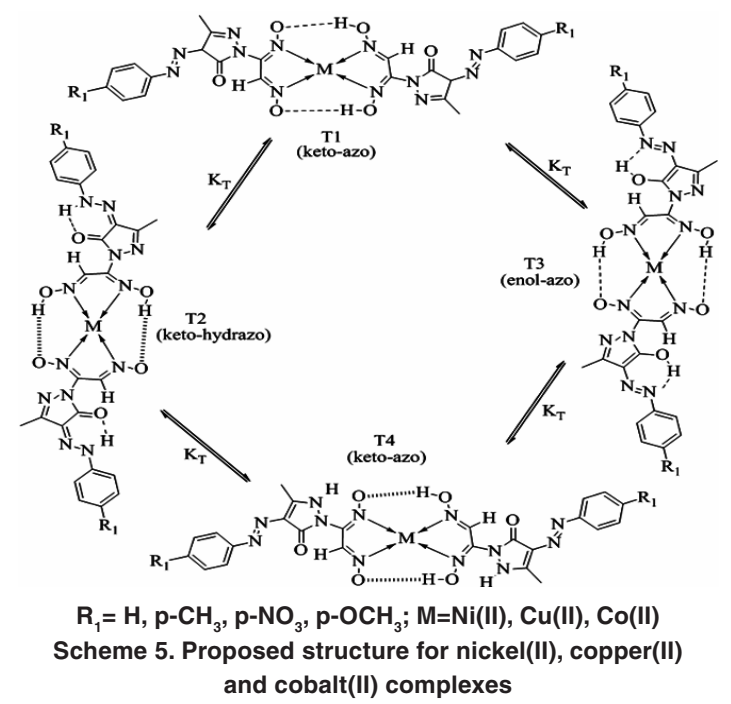

\section{Biological activity}

The synthesized compounds have been examined for their antimicrobial (antibacterial andanti-fungal)activity.

\section{Antimicrobial activity}

The In vitro antimicrobic (S. aureus, $B$. subtilis, E. coli, $P$. aeruginosa and antifungal (C. albicans, C. tropicalis and C. glabrata laboratory isolate) activities of the compounds $1 \mathrm{a}-4 \mathrm{c}$ were evaluated by agar well diffusion method ${ }^{22}$. The results for the antimicrobial study of the tested compounds against the test organisms are illustrated as in Table 9.

Table 9: Antimicrobic activity of the synthesized compounds by well diffusion assay

\begin{tabular}{|c|c|c|c|c|c|c|c|c|c|}
\hline \multirow[t]{2}{*}{ S. No } & \multirow[t]{2}{*}{ Compounds tested } & \multirow[t]{2}{*}{ Effective concentration ( $\mu \mathrm{g} /$ well) } & \multicolumn{7}{|c|}{ Antimicrobic activity of metal complexes [inhibition zone $\mathrm{mm}$ ] } \\
\hline & & & A & B & $\mathrm{C}$ & $\mathrm{D}$ & $\mathrm{E}$ & $\mathrm{F}$ & G \\
\hline 1 & $1 \mathrm{a}$ & 150 & 9 & 9 & 10 & 14 & 14 & 14 & 14 \\
\hline 2 & $1 b$ & 150 & 10 & 9 & 9 & 15 & 14 & 15 & 15 \\
\hline 3 & 1c & 150 & 8 & 8 & 9 & 13 & 14 & 14 & 14 \\
\hline 4 & $2 a$ & 150 & 11 & 10 & 10 & 14 & 14 & 14 & 14 \\
\hline 5 & $2 b$ & 150 & 12 & 11 & 11 & 15 & 15 & 14 & 14 \\
\hline 6 & $2 c$ & 150 & 8 & 9 & 9 & 12 & 13 & 12 & 12 \\
\hline 7 & $3 a$ & 150 & 11 & 10 & 10 & 17 & 17 & 18 & 16 \\
\hline 8 & $3 b$ & 150 & 15 & 14 & 14 & 20 & 19 & 19 & 18 \\
\hline 9 & $3 c$ & 150 & 8 & 8 & 8 & 15 & 14 & 14 & 14 \\
\hline 10 & $4 a$ & 150 & 9 & 8 & 8 & 13 & 15 & 15 & 14 \\
\hline 11 & $4 b$ & 150 & 11 & 10 & 10 & 16 & 16 & 15 & 16 \\
\hline 12 & $4 c$ & 150 & 8 & 8 & 8 & 14 & 14 & 14 & 14 \\
\hline Chloramphenicol & 100 & -- & -- & -- & 30 & 30 & 30 & 30 & \\
\hline Fluconozole & 100 & 28 & 25 & 25 & -- & -- & -- & -- & \\
\hline
\end{tabular}


A-Candida albicans, B-Candida trophicalis, C-Candida glabrata, D-Staphylococcus aureus, E-Bacillus subtilis, F-Escherichia coli, G-Pseudomonas aeruginosa

Results of the synthesized compounds1a, 1b, 1c, 2a, 2b, 2c, 3a, 3b, 3c, 4a, 4b and 4c are outlined for antimicrobic activity with respect to four bacteria Staphylococcus aureus, Bacillus subtilis, Escherichia coli, Pseudomonas aeruginosa and three fungal as Candida albicans, Candida tropicalis and Candida glabrata are illustrated in Table 9.

Antimicrobial activity against "Gram -positive", "Gram-negative"bacteria and agaist fungus have been seen across all the synthesized compounds. Newly synthesized compounds $\mathbf{3 b}$ showed signifant gross broad-spectrum antimicrobial activity, i.e., against "Gram-positive”, "Gram-negative bacteria "as well as anti fungal. The effective concentration of these active compounds was $150 \mu \mathrm{g} /$ well.

\section{CONCLUSION}

Investigations into the synthesis of new hydrazone oxime ligands having pyrazolone group and their metal complexes have been carried out in this study. The synthesized pyrazolone derivatives of $\mathrm{Co}(\mathrm{II}), \mathrm{Ni}(\mathrm{II})$ and $\mathrm{Cu}(\mathrm{II})$ complexes were isolated and their structures were characterized using physicochemical techniques. Also, tautomerism in the ligands was investigated by spectroscopic.Chemical propertirs of the complex compounds depend upon Tautomerism and also physical properties like colour fastness depend on it. The attachment of ligands to the metal ion in a neutral bidentate form with the azomethine nitrogen $(\mathrm{C}=\mathrm{N})$ and the carbonyl oxygen $(\mathrm{C}=\mathrm{O})$. The calculated magnetic moments of the coordinated complex compounds as 2.92 B.M for the nickel(II) and 1.65 B.M for the copper(II) are approximate the spin values only and propose a square planar geometry for the complexes. The compounds 3b exhibited broad-spectrum antimicrobial activity. Expeditions to explore their potentialities in the future for other biological assays are needed to investigate further. Furthermore, some Co(II) complexes act as potent anti-cancer agents due to their anti-proliferative effects; in this regard, the synthesized compounds should be investigated in the future as well. These complexes can be further explored in the dye/color industry due to the presence of chromophores.

\section{ACKNOWLEDGMENT}

We acknowledge to Aydın Adnan Menderes University Scientific Research Foundation for their financial support (Project No: FEF-18022).

\section{Conflict of Interest}

The authors declare that they have no conflict of interest.

\section{REFERENCES}

1. Wang, X. H; Jia, D. Z.; Liang, Y.J.; Yan, S. L.; Ding, Y.; Chen, L. M.; Shi, Z.; Zeng, M. S.; Liu, G. F.; Fu, L. W.; Lgf-YL-9 induces apoptosis in human epidermoid carcinoma KB cells and multidrug resistant KBv200 cells via reactive oxygen species in dependent mitochondrial pathway, Cancer Lett., 2007, 249, 256-270.

2. Kimata, A.; Nakagawa, H.; Ohyama, R.; Fukuuchi, T.; Ohta S.; Dohura, K.; Suzuki, T.; Miyata, N.; New series of antiprion compounds: pyrazolone derivatives have the potent activity of inhibiting protease-resistant prion protein accumulation. J. Med. Chem., 2007, 50(21), 5053-5056.

3. Costa, D.; Marques, A.P.;Reis, R.L.;Lima, J.L.F.C.; Fernandes, E.; Inhibition of human neutrophil oxidative burst by pyrazolone derivatives. Free Radical Biol. Med., 2006, 40, 632-640.

4. Arnost, M.; Pierce, A.; ter Haar, E.; Lauffer, D.;Madden, J.; Tanner, K.; Green, J.; 3-Aryl4-(arylhydrazono)-1H-pyrazol-5-ones: Highly ligand efficient and potent inhibitors of GSK3beta. Bioorg Med Chem Lett., 2010, 20, 1661-1664.

5. Ragavan, R.V.; Vijaykumar, V.; Suchetha, K.N.; Synthesis of some novel bioactive 4-oxy/thio substituted-1H-pyrazol-5(4H)-ones via efficient cross-Claisen condensation. Eur. J. Med. Chem., 2009, 44, 3852-3857.

6. Gursoy, A.; Demirayak, S.; Capan, G.; Erol, K.; Vural, K.; Synthesis and preliminary evaluation of new 5-pyrazolinone derivatives as analgesic agents. Eur. J. Med. Chem., 2000, 35, 359-364.

7. Castagnolo, D.; Manetti, F.; Radi, M.; Bechi, B.; Pagano, M.; Logu, A.D.; Meleddu, R.; Saddi, M.; Botta, M.; Synthesis, biological evaluation, and SAR study of novel pyrazole analogues as inhibitors of Mycobacterium tuberculosis: Part 2. Synthesis of rigid pyrazolones. Bioorg. Med. Chem., 2009,17, 5716-5721. 
8. $\quad$ Parekh, N.; Maheria, K.; Patel, P.; Rathod, M.; Study on antibacterial activity for multidrug resistance stain by using phenyl pyrazolones substituted 3-amino $1 \mathrm{H}$-pyrazolon $(3,4-b)$ quinoline derivative In vitro condition. Int. $\mathrm{J}$. Pharm. Tech Res., 2011, 3, 540-548.

9. Mariappan, G.; Saha, B.P.; Sutharson, L.; Haldar, A.; Synthesis and bioactivity evaluation of pyrazolone derivatives. Indian Journal of Chemistry B., 2010, 49, 1671-1674.

10. Manojkumar, P.; Ravi, T.K.; Subbuchettiar, G.; Synthesis of coumarin heterocyclic derivatives with antioxidant activity and in vitro cytotoxic activity against tumour cells. Acta Pharm., 2009, 59, 159-170.

11. Isloor, A.M.;Kalluraya, B.;Shetty, P.; Regioselective reaction: synthesis, characterization and pharmacological studies of some new Mannich bases derived from 1, 2, 4-triazoles. Eur. J. Med. Chem., 2009, 44, 3784-3787.

12. Isloor, A.M.; Kalluraya, B.; Rao, M.; Sydnone derivatives: part IV; synthesis of 3-aryl4-(substituted pyrazolidene hydrazine-4thiazolyl) sydnones as possible analgesic and anticonvulsant agents. J. Saudi Chem. Soc., 2000, 4, 265- 270.

13. Kalluraya, B.;Isloor, A.M.; Shenoy, S.; Synthesis and biological activity of 6-substituted-3-[4(3-substituted pyrazolidene) hydrazino-4thiazolyl] coumarins. Indian J. Heterocyclic Chem., 2001, 11, 159-162.

14. Sunil, D.; Isloor, A.M.; Shetty, P.; Synthesis, characterization and anticancer activity of 1,2,4Triazolo [3,4-b]-1,3,4-thiadiazoles on Hep G2 cell lines. Der Pharma Chemica., 2009, 1, 19-26.

15. Jensen, B.S.; The synthesis of 1-phenyl-3methyl-4-acyl-pyrazolones-5. Acta Chem. Scand., 1959, 13, 1668-1670.

16. Sarıkavaklı, N.; Irez, G.; Synthesis and Complex Formation of Some Novel vic-Dioxime Derivatives of Hydrazones. Turk J Chem., 2005, 29, 107-115.

17. Elnagdi, M.H.; Elgemeie, G.E.H.; Abdelaal, F.A.E.; Recent developments in the synthesis of pyrazole derivatives. Heterocyclic., 1985, 23, 3121- 53.

18. Sener, N.; Karcı, F.; Sener, I.; Serkan, Y.; Synthesis, Absorption Properties and Biological Evaluation of Some Novel Disazo Dyes Derived from Pyrazole Derivatives. Asian J. Chem., 2015, 27, 3003-3012.

19. Hamama, W.S.; El-Gohary, H.G.; Kuhnert, N.; Zoorob, H.H.; Chemistry of pyrazolinones and their applications. Curr. Org. Chem., 2012, 16, 373-399.

20. Aktan, E.; Ertan, N.; Uyar, T.; Synthesis, characterization and theoretical study of new hetarylazopyrazolone dyes and investigation of their absorption spectra. J. Mol. Struct. 2014, 1060, 215-222.

21. C.Perez.; M.Pauliand P.; Bazerque., Acta. Biol. Med.Exp,1990, 15,113.

22. K. Harathi.; D. Giribabu.; C.Varadarajulu Naidu.; Phytochemical Evaluation and In vitro Antibacterial Activity of Sphaeranthus indicus (L.)-An Important Ant jaundice Medicinal Plant;. American Journal of Plant Sciences., 2017, 8, 5.

23. Ertan, N.; Synthesis of some hetarylazopyrazolone dyes and solvent effects on their absorption spectra. Dyes Pigm., 2000, 44, 41-48.

24. Song, H.; Chen, K.; Tian, H.; Synthesis of novel dyes derived from 1-ethyl-3-cyano-6hydroxy-4-methyl-5-amino-2-pyridone. Dyes Pigm., 2002, 53, 257-262.

25. Sarıkavaklı, N.; Çakıcı, H.T.; Synthesis and Characterization of $\mathrm{Co}$ (II), Ni (II), $\mathrm{Cu}$ (II) and Zn (II) Complexes of Glyoxime Hydrazone. Asian J. Chem., 2011, 23(3), 1321-1326.

26. Seferoglu, Z.; Yalcın, E.; Babur, B.; Seferoglu, N.; Hokelek, T.; Yılmaz, E.; Sahin, E.; Phenylazoindole dyes-Part I: The syntheses, characterizations, crystal structures, quantum chemical calculations and antimicrobial properties. Spectrochim. Acta., 2013, 113, 314-324.

27. Nami, S. A. A.; Husain, A.; Ullah, I.; Self assembled homodinuclear dithiocarbamates: One pot. synthesis and spectral characterization. Spectrochim. Acta Part A., 2014, 118, 380-388.

28. Aktan, E.; Babür, B.; Seferoglu, Z.; Hökelek, T.; Sahin ,E.; Synthesis and structure of a novel hetarylazoindole dye studied by $\mathrm{X}$-ray diffraction, FT-IR, FT-Raman, UV-Vis, NMR spectra and DFT calculations. J. Mol. Struct., 2011, 1002, 113-120.

Nami, S.A.A.; Siddiqi, K.S.; Unique keto-enol tautomerism in transition metal complexes of cyanoimidodithiocarbonate. J. Chem. Res. 2006, 563-565.

29. Pettinari, C.; Marchetti, F.; Cingolani, A.; Gindulyte, A.; Massa , L.; Rossi, M.; Caruso, F.; Syn-anti Conversion in octahedral bis (betadiketonato) diorganotin (IV) derivatives containing fluorinated 4-acyl-5-pyrazolonato donors. Eur.J. Inorg. Chem., 2001, 8, 2171-2180.

30. Mustafa S.K.; Oyouni A. A. A.; Aljohani M.M.H.; and Ahmad M. A.;Polyphenols more than an Antioxidant: Role and Scope.J. Pure Appl. Microbiol., 2020, 14(1), 47-61. 